\title{
Thermodynamics of hydride formation and decomposition in supported sub-10 nm Pd nanoparticles of different sizes
}

\author{
Carl Wadell ${ }^{\mathrm{a}, *}$, Torben Pingel $^{\mathrm{a}}$, Igor Zorića ${ }^{\text {, Vladimir P. Zhdanov }}{ }^{\mathrm{a}, \mathrm{b}}$, \\ Christoph Langhammer ${ }^{\mathrm{a}, *}$ \\ ${ }^{a}$ Department of Applied Physics, Chalmers University of Technology, SE-421 96 Göteborg, \\ Sweden \\ ${ }^{b}$ Boreskov Institute of Catalysis, Russian Academy of Sciences, Novosibirsk 630090, Russia
}

\begin{abstract}
Hydrogen storage properties of supported Pd nanoparticles with average sizes in the range from $2.7 \mathrm{~nm}$ to $7.6 \mathrm{~nm}$ were studied using indirect nanoplasmonic sensing. For each particle size a series of isotherms was measured and, through Van't Hoff analysis, the changes in enthalpy upon hydride formation/decomposition were determined. Contrary to the expected decrease of the enthalpy, due to increasing importance of surface tension in smaller particles, we observe a very weak size dependence in the size range under consideration. We attribute this to a compensation effect due to an increased fraction of hydrogen atoms occupying energetically favorable subsurface sites in smaller nanoparticles.
\end{abstract}

Keywords: Palladium, hydrogen storage, subsurface hydrogen, thermodynamics, isotherms, plasmonic sensing

\section{Introduction}

Pd is an attractive model system for studying hydride formation and decomposition in metals due to its easily feasible hydrogen absorption and release. Since the first report of the hydrogen storing properties of Pd in 1866 [1], a wealth of experimental and theoretical data has been obtained for both phases of absorbed hydrogen (i.e., diluted $\alpha$ phase at low $\mathrm{H}_{2}$ pressure and hydride $\beta$ phase at higher pressure) for bulk [2] and, to a more limited extent, nanostructured systems [3]. The corresponding experimental studies were recently extended down to single $\mathrm{Pd}$ nanoparticles $[4,5]$. When it comes to nanosized entities, the understanding of the $\mathrm{H} / \mathrm{Pd}$ system behaviour is, however, sill limited. In this case, the thermodynamics and kinetics of hydrogen storage are

\footnotetext{
* Corresponding author

Email addresses: carl.wadell@chalmers.se (Carl Wadell), clangham@chalmers.se (Christoph Langhammer)
} 
strongly influenced by surface and interfacial effects as well as by the changes in the electronic structure of the sorption material. The former is the result of an increase of the fraction of surface and interfacial sites for hydrogen absorption compared to that of the bulk sites. In addition, the role of surface tension increases [3]. The relative significance of these factors is still not fully clear.

Most of the published work on the influence of nanosizing below $10 \mathrm{~nm}$ on hydrogen storage thermodynamics is based on measured pressure composition isotherms for surfactant stabilized $\mathrm{Pd}$ nanoparticles $[6,7,8]$. A summary of the corresponding findings includes: a decrease in plateau width (miscibility region), an increase in the slope of the plateau, and a decreased hysteresis between absorption and desorption for smaller particle sizes. However, with the exception of the studies by Yamauchi et al. [9] and Narehood et al. [10], there are no attempts to explore the size dependence on the thermodynamics of the system, by measuring isotherms at different temperatures. In the former case [9], size dependent enthalpy and entropy for hydride formation were obtained for two different sizes $(2.6 \mathrm{~nm}$ and $7.0 \mathrm{~nm})$ of surfactant stabilized Pd nanoparticles from measured isotherms. In the latter case [10], the experimentally obtained isotherms for $2.1 \mathrm{~nm}$ and $3.1 \mathrm{~nm} \mathrm{Pd}$ nanoparticles, stabilized with a carbon coating, indicated narrowing of the two phase region and a lowering of the critical temperature. Herein, we focus on the thermodynamics of the hydride formation and decomposition in supported "clean" (no surfactants or stabilizers) $\mathrm{Pd}$ nanoparticles with the average size ranging from $2.7 \mathrm{~nm}$ to $7.6 \mathrm{~nm}$.

\section{Experimental Methods}

To monitor the hydrogen concentration in the particles of this size, we used indirect nanoplasmonic sensing (INPS) [11]. The INPS chip includes sensor Au nanodisks covered by a nm-thick dielectric $\mathrm{SiO}_{2}$ layer onto which the probed $\mathrm{Pd}$ particles are deposited as schematically shown in Figure 1(a). A side view of the real sensor structure as seen in SEM is displayed in Figure 1(b). The $\mathrm{Au}$ nanodisks, $140 \mathrm{~nm}$ diameter and $20 \mathrm{~nm}$ high, were fabricated by holemask colloidal lithography [12] and subsequently covered by a $10 \mathrm{~nm}$ sputtered $\mathrm{SiO}_{2}$ layer. The $\mathrm{Pd}$ nanoparticles were deposited on top of the $\mathrm{SiO}_{2}$ layer by evaporating a small amount of Pd (2 - $10 \AA$ nominal film thickness) followed by a $36 \mathrm{~h}$ annealing at $400^{\circ} \mathrm{C}$ in air and a shorter $2 \mathrm{~h}$ annealing at $600^{\circ} \mathrm{C}$ in air. This thermal treatment combined with poor wetting properties of $\mathrm{Pd}$ on the $\mathrm{SiO}_{2}$ surface drives the formation of $\mathrm{Pd}$ nanoparticles (their size can be varied by varying the amount of deposited $\mathrm{Pd}$ ). The INPS method relies on measuring the $\mathrm{Pd}$-induced changes in the plasmon-mediated light absorbance of the $\mathrm{Au}$ nanodisks as schematically illustrated in Figure 1(c). As previously shown [11], the changes of the plasmon peak position $\left(\Delta \lambda_{\text {centroid }}\right)$, peak height $(\Delta \mathrm{Ext})$, and full width at half maximum of the peak $(\Delta \mathrm{FWHM})$ are all proportional to the hydrogen concentration in the sensed nanoparticles ( $\mathrm{Pd}$ in our case).

The size distribution of the $\mathrm{Pd}$ nanoparticles was determined by TEM imaging after completion of the hydrogen storage measurements, using the above 
described process, on $\mathrm{Si}_{3} \mathrm{~N}_{4}$ TEM windows with a $\mathrm{SiO}_{2}$ surface layer in a similar fashion as was done earlier [13]. The mean diameter of a particular particle distribution can be obtained directly from TEM images using the projected area diameters. One can also calculate the mean particle diameter from an average nanoparticle volume by assuming a spherical nanoparticle shape. The latter values are what we have used when the nanoparticle size is reported below. To investigate the validity of the assumption of spherical particles, tilted TEM images were employed. In order to get side views, the particles were deposited on $\mathrm{Si}_{3} \mathrm{~N}_{4}$ TEM windows covered by $\mathrm{SiO}_{2}$ nanocones (for details, see the Supporting Information). In this way, by tilting the window $30^{\circ}$, side views of the particles on the sides of the cones were obtained (Figure 2). As one can see, the smaller particles tend to be single crystals and take on a spherical shape whereas the larger ones are poly-crystalline and take more complex shapes.

The hydrogen storage measurements were carried out in a small stainless steel vacuum cell (volume $\sim 20 \mathrm{~cm}^{3}$ and a base pressure of $\sim 10^{-4} \mathrm{mbar}$ ). The cell is equipped with two sapphire glass windows enabling continuous optical transmission measurements on the INPS chip in order to map out the plasmonic resonance of the sensing nanoparticle during the $\mathrm{H}_{2}$ exposure. The $\mathrm{H}_{2}$ pressure in the cell, as monitored by a capacitance pressure gauge, was increased/decreased slowly during absorption/desorption measurements. A series of absorption and desorption measurements was carried out with different pressure increase and decrease rates (see the Supporting Information) to make sure that we have an equilibrium situation in the cell. The absorption and desorption isotherms were obtained by following changes of $\Delta \lambda_{\text {centroid }}$ or $\Delta$ Ext. Due to variations in the coupling between the sensor $\mathrm{Au}$ particles and the sensed $\mathrm{Pd}$ particles as Pd particle sizes vary the different characteristics for the sensor proves to have different sensitivity. For the smallest particles $\Delta$ Ext is more sensitive to the hydride formation than $\Delta \lambda_{\text {centroid }}$, whereas for the larger particles the opposite is true. Therefore, for each $\mathrm{Pd}$ particle size, the Au plasmonicresonance feature that gave the best signal to noise ratio was chosen.

Figure 3 shows an example of a single hydrogen absorption isotherm for Pd nanoparticles, with an average diameter of $3.9 \mathrm{~nm}$, measured by INPS at $40^{\circ} \mathrm{C}$ with $\Delta$ Ext used as readout proportional to the concentration of absorbed hydrogen, $C$. The enthalpy and entropy changes, $\Delta H$ and $\Delta S$, upon hydride formation/decomposition are obtained by identifying the plateau pressure, i.e. the region in the $p-\Delta$ Ext diagram where the three phases $(\alpha, \beta$, and gas phase) are in equilibrium. Once the plateau pressures are identified for a series of isotherms, $\Delta H$ and $\Delta S$ are determined from the requirement that the chemical potentials of the gas and hydride phase are equal in equilibrium, or

$$
\ln \left(\frac{P}{P_{0}}\right)=\frac{\Delta H}{k_{\mathrm{B}} T}-\frac{\Delta S}{k_{\mathrm{B}}}
$$

where $P$ is equilibrium plateau $\mathrm{H}_{2}$ pressure, and $P_{0}=1013 \mathrm{mbar}$ (note that $\Delta H$ and $\Delta S$ are here per $\mathrm{H}_{2}$ molecule). Specifically, $\Delta H$ and $\Delta S$ can be extracted from the Van't Hoff plots (where $\ln \left(P / P_{0}\right)$ vs. $1 / T$ is plotted from a series of isotherms) as the slope and intercept of the linear fit of the data points. 
Since we do not observe a constant plateau in the miscibility region but instead a sloped plateau, as has been earlier reported for nanosized Pd [14], the determination of the plateau pressure was done as illustrated in Figure 3. Specifically, each isotherm was approximated by three lines within the miscibility region, and the midpoint of the two intercepts was chosen as the plateau pressure for a given isotherm. Similar procedures have been employed by others previously $[15,16,17]$.

\section{Results and Discussion}

A series of absorption and desorption isotherms, measured in the temperature interval from $30^{\circ} \mathrm{C}$ to $80^{\circ} \mathrm{C}$, in steps of $10^{\circ} \mathrm{C}$, for two different particle sizes, $2.7 \mathrm{~nm}$ and $5.3 \mathrm{~nm}$, are shown in Figure 4 (middle). The TEM images and size distributions for the two samples are shown as well (left panels). The corresponding Van't Hoff plots are presented (right panels) both for absorption and desorption. We observe an excellent fit to the expected linear relation in the $\ln \left(P / P_{0}\right)$ vs. $1 / T$ diagram. The shift between the two straight lines in the Van't Hoff diagram is the consequence of the absorption-desorption hysteresis. It shrinks the smaller the particles are, as reported and explained earlier [18]. Similar measurements were carried out for a series of other Pd nanoparticle sizes (see the Supporting Information for the complete set of isotherms) and the corresponding values of $\Delta H_{\alpha \rightarrow \beta}, \Delta S_{\alpha \rightarrow \beta}, \Delta H_{\beta \rightarrow \alpha}, \Delta S_{\beta \rightarrow \alpha}$ together with the particle mean diameter and the standard deviation (Std) of the particle size distribution are shown in Table 1. Surprisingly, the obtained thermodynamic variables appear to be rather independent of the nanoparticle size.

In Figure 5, the enthalpy changes upon hydride formation are shown as a function of nanoparticle size. To interpret these data, we note that at the nanoscale one of the key factors resulting in the dependence of the thermodynamics on the particle size is surface tension. Employing the conventional Kelvin model, one can show (see, e.g., [19]) that the corresponding contribution to the enthalpy change (per $\mathrm{H}$ atom) is $-2 \gamma v / R$, where $\gamma$ is the surface tension $\left(0.2 \mathrm{eV} / \AA^{2}\right.$ for $\left.\mathrm{Pd}\right), v$ is the partial volume of hydrogen in the hydride phase $(v$ $=2.6 \AA^{3}$ ), and $R$ is the particle radius. Physically, this classical effect is related to a decrease of the $\mathrm{H}$ binding energy with compression of $\mathrm{Pd}$ (reviewed in [20]; a similar effect takes often place in other cases, e.g., for $\mathrm{CO}$ adsorption on $\mathrm{Pd}$ nanoparticles [21]). For nanoparticles, the compression is due to surface tension. For Pd nanoparticles, experiment and DFT calculations indicate (reviewed in [22]) that this compression is accurately described by the conventional model down to sizes smaller than in our case. Thus, one can expect to observe an appreciable decrease of the observed enthalpy change with decreasing particle size (see the dashed line in Figure 5). The absence of the decrease in the experiment indicates that the effect of surface tension is somehow compensated. We believe that the compensation is related to the increasing importance of subsurface sites with decreasing particle size.

In $\mathrm{Pd}$, the subsurface sites are known to have higher absorption energies compared to the bulk sites $[23,24]$. The adsorption energy at surface sites is 
in turn even higher than that corresponding to the subsurface sites (see, e.g., [25] and references therein). The surface sites are therefore expected to be filled during measurements of the enthalpy and entropy upon hydride formation/decomposition. In contrast, the population of the subsurface sites may change and this is known to be one of the reasons for the observed shrinking of the miscibility gap in the phase diagram with decreasing particle size [7]. In what follows, we formulate a minimal generic analytical model showing that the presence of the subsurface sites may also compensate the contribution of surface tension to the enthalpy change.

We are interested in describing equilibrium between hydrogen in the gas phase and Pd. According to thermodynamics, as already noted, the conditions for equilibrium can be formulated in terms of chemical potential for different phases. The chemical potential of $\mathrm{H}_{2}$ molecules in the gas phase can be expressed as $[26]$

$$
\mu_{\mathrm{H}_{2}}=k_{\mathrm{B}} T \ln \left[\frac{P}{\left(k_{\mathrm{B}} T\right)^{5 / 2}}\left(\frac{2 \pi \hbar^{2}}{m}\right)^{3 / 2}\right],
$$

where $P$ and $T$ are pressure and temperature, and $m$ is the mass of a $\mathrm{H}_{2}$ molecule. This expression takes only the translation partition function into account. The contribution of rotation and especially vibrations to $\mu_{\mathrm{H}_{2}}$ is minor and can be neglected for our goals.

A Pd nanoparticle can be viewed as a sphere with a central "bulk" volume and an outer shell containing the subsurface sites (the surface sites are considered to be occupied by $\mathrm{H}$ and are not involved into the analysis). The volumes of these two regions are, respectively, $V_{\mathrm{b}}=(4 \pi / 3)(R-h)^{3}$ and $V_{\mathrm{ss}}=$ $(4 \pi / 3)\left[R^{3}-(R-h)^{3}\right]$, where $R$ is the particle radius, and $h \simeq 3 \AA$ is the thickness of the subsurface volume. The fractions of the sites located there are, respectively, $f_{\mathrm{b}}=V_{\mathrm{b}} / V_{\mathrm{t}}$ and $f_{\mathrm{ss}}=V_{\mathrm{ss}} / V_{\mathrm{t}}$, where $V_{\mathrm{t}}=(4 \pi / 3) R^{3}$ is the total particle volume.

Taking into account that the experiments were performed at temperatures and particle sizes where the hysteresis loops are narrow, we ignore the plateautype features and describe the $\mathrm{H}$ absorption in $\mathrm{Pd}$ by using the simplest Langmurian-type model. In particular, the chemical potential of $\mathrm{H}$ atoms in the bulk hydride is represented as

$$
\mu_{\mathrm{b}}^{\mathrm{H}}=-E_{0}+k_{\mathrm{B}} T \ln \left[\theta_{\mathrm{b}} /\left(1-\theta_{\mathrm{b}}\right)\right]+2 \gamma v / R,
$$

where $\theta_{\mathrm{b}}$ is the $\mathrm{H}$ coverage of the bulk sites, and $E_{0}$ is the gain in energy during the hydride formation at $R \rightarrow \infty$ (in terms of the nearest-neighbor interactions between $\mathrm{H}$ atoms, $\epsilon_{1}$, we have $E_{0}=E_{\alpha}+z \epsilon_{1} / 2$, where $E_{\alpha}$ is the gain in energy in the $\alpha$ phase, and $z$ is the number of the nearest-neighbor sites).

The chemical potential of $\mathrm{H}$ atoms in the subsurface volume is represented as

$$
\mu_{\mathrm{ss}}^{\mathrm{H}}=-E_{0}-\Delta E+k_{\mathrm{B}} T \ln \left[\theta_{\mathrm{ss}} /\left(1-\theta_{\mathrm{ss}}\right)\right]+2 \gamma v / R,
$$

where $\Delta E>0$ is the energy difference between the bulk and sub-surface sites, and $\theta_{\mathrm{ss}}$ is the $\mathrm{H}$ coverage of the subsurface sites. 
Concerning equations (2) and (3), we note that, in principle, one can extend them in the framework of the mean-field approximation describing the plateau-type features in the absorption isotherms. This approximation is, however, known to overestimate the role of these features (for a cubic lattice with nearest-neighbour interactions, e.g., the mean-field expression for the critical temperature is $k_{\mathrm{B}} T_{\mathrm{c}}=1.5 \epsilon_{1}$, while the accurate one is $\left.k_{\mathrm{B}} T_{\mathrm{c}}=1.128 \epsilon_{1}[27]\right)$. Taking also into account that the corresponding equations are cumbersome and not convenient for general readership, we believe that the extension of the analytical treatment will only obscure the main message. For these reasons, we keep our analysis on the simplest level allowing us to explain our findings.

At equilibrium, we have

$$
\mu_{\mathrm{H}_{2}}=2 \mu_{\mathrm{b}}^{\mathrm{H}}=2 \mu_{\mathrm{ss}}^{\mathrm{H}} .
$$

These conditions in combination with expressions (1) - (3) allow us to calculate $\theta_{\mathrm{b}}$ and $\theta_{\mathrm{ss}}$ as a function of $\ln \left(P / P_{0}\right), E_{0}$, and $\Delta E$. The average coverage is then given by

$$
\langle\theta\rangle=f_{\mathrm{b}} \theta_{\mathrm{b}}+f_{\mathrm{ss}} \theta_{\mathrm{ss}} .
$$

For given $T$ and $R$, in analogy with the experiment, the pressure corresponding to the hydride formation can be identified with that corresponding to $\langle\theta\rangle=0.5$. Performing such calculations at different $T$, one can construct the Van't Hoff plot and get the apparent $\Delta H$ and $\Delta S$ for the hydride formation at a given $R$. Performing then the calculations at different $R$, one can obtain the dependence of $\Delta H$ and $\Delta S$ on $R$.

With decreasing $R$, the apparent enthalpy of hydride formation decreases due to surface tension and increases due to increasing fraction of the subsurface sites. If these effects are comparable, the dependence on $R$ may be weak. To describe this case, we formally have two free parameters, $E_{0}$ and $\Delta E$. We fix $E_{0}$ at $17 \mathrm{~kJ} /(\mathrm{mol} \mathrm{H})$, to reproduce $\Delta H$ for a measurement carried out on a $30 \mathrm{~nm}$ thick Pd film, see Table 1, at large particle sizes. The only remaining parameter, $\Delta E$, is selected to be $9 \mathrm{~kJ} /(\mathrm{mol} \mathrm{H})$. Concerning the values chosen for the model, the experimental value for $E_{\alpha}$, reported in the literature, is $10.3 \mathrm{~kJ} /(\mathrm{mol} \mathrm{H})$ [28]. This value is obtained from the measurements in the $\alpha$ phase (low $\mathrm{H}$ concentration). The value of $E_{0}$ should be higher in the $\beta$ phase. From the known critical temperature of the system $\left(292^{\circ} \mathrm{C}[29]\right)$, one can easily estimate that the contribution of the $\mathrm{H}-\mathrm{H}$ interactions to $E_{0}$ is about $10 \mathrm{~kJ} /(\mathrm{mol}$ $\mathrm{H})$, and accordingly $E_{0}$ is expected to be about $20 \mathrm{~kJ} /(\mathrm{mol} \mathrm{H})$. Thus, the value we use, $17 \mathrm{~kJ} /(\mathrm{mol} \mathrm{H})$, is reasonable. The chosen value for $\Delta E$ appears to be reasonable as well (see, e.g., the analysis of the corresponding TPD data [25]).

The model predictions obtained with the parameters above are shown in Figure 5 as a solid line. The dashed line corresponds to the case with $\Delta E=0$ when the contribution of the subsurface sites is the same as that of the bulk sites, and, accordingly, the effect of surface tension is not compensated. With $\Delta E=9 \mathrm{~kJ} /(\mathrm{mol} \mathrm{H})$, the model is seen to reproduce the experimentally observed very weak size dependence of $\Delta H$ in the investigated size range. The value of $\Delta H$ is, however, predicted to be slightly lower than in the experiment. If the 
contribution for the rotational partition function is included in Eq. (1) the shape of the $\Delta H$ curve is not noticeably affected. However, it results in a shift of the entire curve by $+2.7 \mathrm{~kJ} / \mathrm{mol}$ which can be compensated by decreasing the value of $E_{0}$ to $15.6 \mathrm{~kJ} /(\mathrm{mol} \mathrm{H})$. Therefore this is not the reason for the difference in the values between the model and the experiment. The difference seems, as discussed below, to be related to the parametrization of $E_{0}$ using the results obtained for the film.

Concerning the values of $\Delta H\left(42-43 \mathrm{~kJ} /\left(\mathrm{mol} \mathrm{H}_{2}\right)\right)$ obtained in our measurements, we note that the corresponding values reported earlier for the bulk $(39-41 \mathrm{~kJ} / \mathrm{mol}[30])$ and thin films $(41 \mathrm{~kJ} / \mathrm{mol}[31])$ are somewhat lower. To confirm the accuracy of our technique, we have therefore performed measurements for a $30 \mathrm{~nm}$ thick Pd film using our setup and studying the optical transmission through the film (the same technique as presented in [31]). The corresponding results, shown in Table 1 (for the isotherms and Van't Hoff analysis, see the Supporting Information), are in good agreement with those reported earlier $[30,31]$. This is indicative that the difference between our values of $\Delta H$ for the nanoparticles and those reported for the bulk has some real physical background. One of the likely explanations is related to the specifics of the measurements of $\Delta H$. For the bulk, $\Delta H$ was typically measured under conditions of appreciable absorption-desorption hysteresis [30]. With decreasing temperature, the role of the hysteresis increases, which might result in an appreciable systematic error in the measured $\Delta H$ as we will show.

At the critical temperature hysteresis should have vanished by definition. However, using the results from [30], one can see that the hysteresis will never be zero for finite temperatures. Hence, one can not simply say that the "true" value of $\Delta H$ will be in the range between $\Delta H_{\alpha \rightarrow \beta}$ and $\Delta H_{\beta \rightarrow \alpha}$ (as is usually expected assuming that the "true" coexistence line is approximately located in the middle of the $\alpha \rightarrow \beta$ and $\beta \rightarrow \alpha$ lines) but it may in fact be outside this range. The situation for particles is different. As particles get smaller the hysteresis shrinks and thus also the uncertainty introduced in the determination of $\Delta H$ by the hysteresis decreases. It is therefore useful to estimate this uncertainty range for bulk Pd by using the results for our film measurement and the reported value for the critical temperature $\left(292^{\circ} \mathrm{C}\right.$ [29]) by assuming that we do not know how the hysteresis shrinks but that it should have shrunk to zero at the critical temperature. A sketch of the procedure can be seen in Figure 6. The maximum value of $\Delta H$ is determined in the Van't Hoff plot from the slope of a line between the point where the absorption line crosses the critical temperature and the point on the desorption line at the lowest temperature used in the measurement. The minimum is similarly determined but using the crossing between the desorption line and the critical temperature and the point of the absorption line at the lowest measured temperature instead. The error margins in the fits of the absorption and desorption lines are also taken into account. From this analysis we get a range of $\Delta H$ that is $37.0-44.7 \mathrm{~kJ} / \mathrm{mol}$. In Figure $\mathbf{5}$ this range is marked with a green area and we note that our measurements for the smaller particles indeed fall in this range. Carrying out the same analysis using the results reported in [30], one obtains the range $35.1-41.3 \mathrm{~kJ} / \mathrm{mol}$. 
This analysis thus emphasizes that the uncertainty in bulk enthalpy of hydride formation is of the order of almost $1 \mathrm{~kJ} / \mathrm{mol}$ outside the range between $\Delta H_{\alpha \rightarrow \beta}$ and $\Delta H_{\beta \rightarrow \alpha}$. A fact that generally has to be kept in mind when quoting such numbers.

Comparing our results with those obtained by Yamauchi et al. [9] where significant size dependence for $\Delta H$ and $\Delta S$ was reported for two different sizes of Pd nanoparticles $(31.0 \mathrm{~kJ} / \mathrm{mol}$ for $2.6 \mathrm{~nm}$ particles and $34.6 \mathrm{~kJ} / \mathrm{mol}$ for $7.0 \mathrm{~nm}$ particles), we note that in their work polymer coated nanoparticles, synthesized using a wet chemical process, were used, whereas our particles have clean surfaces. On that account, it is known that surfactants/polymers on the nanoparticle surface influence hydrogenation properties [32] and accordingly may change the energetics of surface and subsurface sites. Moreover, the particles used in [9] were not annealed and may therefore have size-dependent shapes. This contrasts our work were we annealed at $600^{\circ} \mathrm{C}$ to induce a particle shape close to the equilibrium shape predicted by the Wulff rule (Figure 2). This difference between the two systems implies different $\Delta H$ according to our model since the compensation effect might be different for particles deviating from the spherical shape. Finally, the isotherms for the $2.6 \mathrm{~nm}$ particles in [9] are not reversible at low temperatures. It is therefore difficult to determine whether or not there is hysteresis present for this size. As we discussed above, the presence of a wide hysteresis significantly increases the uncertainty of the derived $\Delta H$ values. Thus, in summary, we believe that the observed differences in the sizedependence of the thermodynamic properties between our work and Yamauchi et al. is a combination of different particle shape, clean vs. surfactant/polymer covered surface, and irreversible isotherms for the smallest particle size complicating the analysis of the thermodynamic parameters in their case. This clearly highlights the importance of detailed knowledge of such parameters if the hydrogenation properties of nanosized systems should be assessed and compared.

\section{Conclusion}

We have used indirect nanoplasmonic sensing to investigate the size dependence of $\Delta H$ and $\Delta S$ upon hydride formation/decomposition in supported $\mathrm{Pd}$ nanoparticles with average sizes in the range from $2.7 \mathrm{~nm}$ to $7.6 \mathrm{~nm}$. Contrary to what we expected, we did not observe any discernible size dependence of $\Delta H$. We attribute this finding to two competing factors: (i) increasing surface strain, which acts to destabilize the hydride, i.e. lower $\Delta H$, and (ii) an increasing fraction of hydrogen atoms occupying subsurface sites, with higher absorption energies compared to bulk sites, which leads to higher $\Delta H$. The "compensation" effect is illustrated by using a generic analytical model with physically reasonable parameters for Pd. Basically, our analysis thus indicates that the enthalpy and entropy of hydride formation/decomposition measured for metal nanoparticles is, in fact, "apparent", because it may depend on the interplay of hydride formation/decomposition and $\mathrm{H}$ absorption and/or adsorption at subsurface sites. Consequently, the fact that $\Delta H$ is nearly independent of size in the size range under consideration does not mean that other features, 
e.g., the hydrogen absorption and hydride decomposition kinetics are not size dependent. In fact, our previous experiments [33] clearly showed that these kinetics are size-dependent, and it can be explained taking among other factors the surface tension into account.

\section{Acknowledgement}

The work was funded by the Swedish Foundation for Strategic Research Framework Program RMA11-0037, the Formas project 229-2009-772, and the Swedish Research Council project 2010-4041. The authors also gratefully acknowledge the funding from the Knut and Alice Wallenberg Foundation and the Swedish Research Council for the advanced transmission electron microscopes and the Knut and Alice Wallenberg Foundation for their support of the $\mu$-fab cleanroom infrastructure in Sweden.

\section{Supporting Information Available}

TEM images, size distributions, isotherm measurements and Van't Hoff analyses for all samples. Size dependence of $\Delta H$ predicted by the model with the values for $E_{0}$ and $\Delta E$ different compared to those used in the main text. Deorption isotherms measured at different pumping rates.

\section{References}

[1] Graham, T., On the Absorption and Dialytic Separation of Gases by Colloid Septa. Phil. Trans. R. Soc. Lond. 1866, 156, 399-439

[2] Manchester, F. D.; A., S.-M.; Pitre, J. M., The H-Pd (Hydrogen-Palladium) System. J. Phase Equilib. 1994, 15 (1), 62-83.

[3] Bérubé, V.; Radtke, G.; Dresselhaus, M.; Chen, G., Size Effects on the Hydrogen Storage Properties of Nanostructured Metal Hydrides: A Review. Int. J. Energ. Res. 2007, 31 (6-7), 637-663.

[4] Liu, N.; Tang, M. L.; Hentschel, M.; Giessen, H.; Alivisatos, A. P., Nanoantenna-Enhanced Gas Sensing in a Single Tailored Nanofocus. Nat. Mater. 2011, 10 (8), 631-636.

[5] Shegai, T.; Langhammer, C., Hydride Formation in Single Palladium and Magnesium Nanoparticles Studied By Nanoplasmonic Dark-Field Scattering Spectroscopy. Adv. Mater. 2011, 23 (38), 4409-4414.

[6] Pundt, A.; Sachs, C.; Winter, M.; Reetz, M. T.; Fritsch, D.; Kirchheim, R., Hydrogen Sorption in Elastically Soft Stabilized Pd-Clusters. J. Alloy. Compd. 1999, 293, 480-483. 
[7] Sachs, C.; Pundt, A.; Kirchheim, R.; Winter, M.; Reetz, M. T.; Fritsch, D., Solubility of Hydrogen in Single-Sized Palladium Clusters. Phys. Rev. $B$ 2001, 64 (7).

[8] Pundt, A.; Suleiman, M.; Bähtz, C.; Reetz, M. T.; Kirchheim, R.; Jisrawi, N. M., Hydrogen and Pd-Clusters. Mat. Sci. Eng. B 2004, 108 (1-2), 19-23.

[9] Yamauchi, M.; Ikeda, R.; Kitagawa, H.; Takata, M., Nanosize Effects on Hydrogen Storage in Palladium. J. Phys. Chem. C 2008, 112 (9), 32943299 .

[10] Narehood, D. G.; Kishore, S.; Goto, H.; Adair, J. H.; Nelson, J. A.; Gutierrez, H. R.; Eklund, P. C., X-Ray Diffraction and H-Storage in Ultra-Small Palladium Particles. Int. J. Hydrogen Energ. 2009, 34 (2), 952-960.

[11] Langhammer, C.; Larsson, E. M.; Kasemo, B.; Zoric, I., Indirect Nanoplasmonic Sensing: Ultrasensitive Experimental Platform for Nanomaterials Science and Optical Nanocalorimetry. Nano Lett. 2010, 10 (9), 3529-3538.

[12] Fredriksson, H.; Alaverdyan, Y.; Dmitriev, A.; Langhammer, C.; Sutherland, D. S.; Zäch, M.; Kasemo, B., Hole-Mask Colloidal Lithography. Adv. Mater. 2007, 19 (23), 4297-4302.

[13] Larsson, E. M.; Millet, J.; Gustafsson, S.; Skoglundh, M.; Zhdanov, V. P.; Langhammer, C., Real Time Indirect Nanoplasmonic in Situ Spectroscopy of Catalyst Nanoparticle Sintering. Acs Catal. 2012, 2 (2), 238-245.

[14] Salomons, E.; Griessen, R.; De Groot, D. G.; Magerl, A., SurfaceTension and Subsurface Sites of Metallic Nanocrystals Determined from H-Absorption. Europhys. Lett. 1988, 5 (5), 449-454.

[15] Kirchheim, R.; Mütschele, T.; Kieninger, W.; Gleiter, H.; Birringer, R.; Koblé, T. D., Hydrogen in Amorphous and Nanocrystalline Metals. Mater. Sci. Eng. 1988, 99, 457-462.

[16] Foo, C. H.; Hong, C. T.; Yeh, C. T., Effect of Pd-Tio2 Interaction on the Enthalpy of Hydrogen Absorption. J. Chem. Soc. Farad. T. 1 1989, 85, 65-70.

[17] Vons, V. A.; Leegwater, H.; Legerstee, W. J.; Eijt, S. W. H.; Schmidt-Ott, A., Hydrogen Storage Properties of Spark Generated Palladium Nanoparticles. Int. J. Hydrogen Energ. 2010, 35 (11), 5479-5489.

[18] Langhammer, C.; Zhdanov, V. P.; Zoric, I.; Kasemo, B., Size-Dependent Hysteresis in the Formation and Decomposition of Hydride in Metal Nanoparticles. Chem. Phys. Lett. 2010, 488 (1-3), 62-66.

[19] Zhdanov, V. P., Effect of Lattice Strain on the Kinetics of Hydride Formation in Metal Nanoparticles. Chem. Phys. Lett. 2010, 492 (1-3), 77-81. 
[20] Larche, F. C., The role of stresses on phase transformations, in: Embury, J.D.; Purdy, G. R. (Eds.), Advances in Phase Transitions; Pergamon: Oxford, 1988, pp. 193-203.

[21] Yudanov, I. V.; Genest, A.; Schauermann, S.; Freund, H. J.; Rösch, N., Size Dependence of the Adsorption Energy of CO on Metal Nanoparticles: A DFT Search for the Minimum Value. Nano Lett. 2012, 12, 2134-2139.

[22] Yudanov, I. V.; Genest, A.; Rösch, N., DFT Studies of Palladium Model Catalysts: Structure and Size Effects. J. Clust. Sci. 2011, 22, 433-448.

[23] Behm, R. J.; Penka, V.; Cattania, M. G.; Christmann, K.; Ertl, G., Evidence for Subsurface Hydrogen on $\operatorname{Pd}(110)$ - an Intermediate Between Chemisorbed and Dissolved Species. J. Chem. Phys. 1983, 78 (12), 74867490 .

[24] Okuyama, H.; Siga, W.; Takagi, N.; Nishijima, M.; Aruga, T., Path and Mechanism of Hydrogen Absorption at $\operatorname{Pd}(100)$. Surf. Sci. 1998, 401 (3), 344-354.

[25] Zhdanov, V. P.; Kasemo, B., Kinetics of the formation of a new phase in nanoparticles. Chem. Phys. Lett. 2008, 460, 158-161.

[26] Landau, L. D.; Lifshitz, E. M., Statistical Physics; Pergamon: Oxford, 1993.

[27] Argyrakis, P.; Groda, Y. G.; Bokun, G. S.; Vikhrenko, V. S., Thermodynamics and diffusion of a lattice gas on a simple cubic lattice. Phys. Rev. E 2001, 64, 066108 .

[28] Clewley, J. D.; Curran, T.; Flanagan, T. B.; Oates, W. A., Thermodynamic Properties of Hydrogen and Deuterium Dissolved in Palladium at Low Concentrations over a Wide Temperature Range. J. Chem. Soc. Farad. T. 1 1973, 69 (2), 449-458.

[29] Wicke, E.; Brodowsky, H.; Züchner, H., Hydrogen in palladium and palladium alloys, in: Alefeld, G.; Völkl, J. (Eds.), Hydrogen in Metals II; Springer Berlin Heidelberg, 1978, pp. 73-155.

[30] Lässer, R.; Klatt, K. H., Solubility of hydrogen isotopes in palladium. Phys. Rev. B 1983, 28, 748-758.

[31] Gremaud, R.; Slaman, M.; Schreuders, H.; Dam, B.; Griessen, R., An optical method to determine the thermodynamics of hydrogen absorption and desorption in metals. Appl. Phys. Lett. 2007, 91, 231916.

[32] Suleiman, M.; Faupel, J.; Borchers, C.; Krebs, H.-U.; Kirchheim, R.; Pundt, A., Hydrogen absorption behaviour in nanometer sized palladium samples stabilised in soft and hard matrix. J. Alloys Compd. 2005 (404406), 523-528. 
[33] Langhammer, C.; Zhdanov, V. P.; Zoric, I.; Kasemo, B., Size-dependent kinetics of hydriding and dehydriding of Pd nanoparticles. Phys. Rev. Lett. 2010, 104, 135502 . 
Table 1: Size characteristics and thermodynamic parameters for Pd nanoparticles annealed at $600{ }^{\circ} \mathrm{C}$. The errors indicated for the values of $\Delta H$ and $\Delta S$ signify a $95 \%$ confidence interval from the linear fit in the Van't Hoff analysis.

\begin{tabular}{|c|c|c|c|c|c|c|}
\hline \multirow{2}{*}{$\begin{array}{c}\text { Mean } \\
\text { diameter } \\
{[\mathrm{nm}]}\end{array}$} & \multirow{2}{*}{$\begin{array}{c}\text { Std } \\
{[\mathrm{nm}]}\end{array}$} & \multirow{2}{*}{$\begin{array}{c}\text { Diameter } \\
\text { from mean } \\
\text { volume }[\mathrm{nm}]\end{array}$} & \multicolumn{2}{|c|}{$\Delta H\left[\mathrm{~kJ} /\left(\mathrm{mol} \mathrm{H}_{2}\right)\right]$} & \multicolumn{2}{|c|}{$\left.\Delta S\left[\mathrm{~J} /(\mathrm{K} \mathrm{mol} \mathrm{H})_{2}\right)\right]$} \\
\hline & & & $\alpha \rightarrow \beta$ & $\beta \rightarrow \alpha$ & $\alpha \rightarrow \beta$ & $\beta \rightarrow \alpha$ \\
\hline 2.59 & 0.62 & 2.73 & $42.6 \pm 1.9$ & $43.4 \pm 2.2$ & $108.2 \pm 5.8$ & $109.6 \pm 6.8$ \\
\hline 3.69 & 0.97 & & 0.9 & 43.4 & \pm 2.8 & $108.7 \pm$ \\
\hline 4.76 & 1.68 & 5.3 & $42.2 \pm 1.2$ & $42.9 \pm$ & $107.4 \pm 3.7$ & $107.8 \pm 5.9$ \\
\hline 6.21 & 3.11 & 7.62 & $42.6 \pm 0.6$ & $45.2 \pm 1.9$ & $107.0 \pm 2.0$ & $112.5 \pm 5.8$ \\
\hline \multicolumn{3}{|c|}{ Film $(30 \mathrm{~nm})$} & $38.9 \pm 1.3$ & $42.6 \pm 1.0$ & $98.4 \pm 4.1$ & $103.6 \pm 2.9$ \\
\hline
\end{tabular}



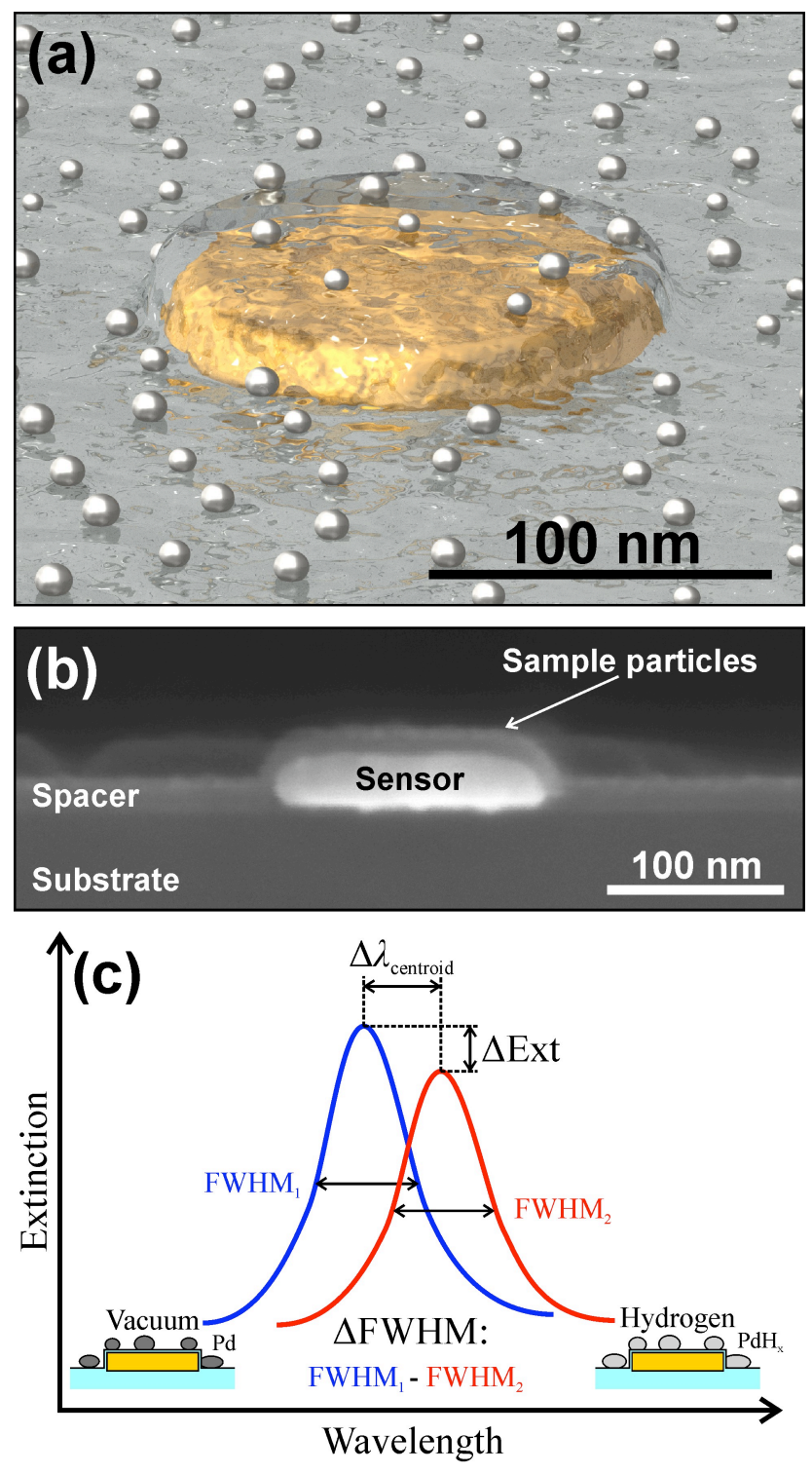

Figure 1: Indirect nanoplasmonic sensing: (a) a schematic drawing of the sensor structure containing gold sensor nanodisks covered by a $\mathrm{SiO}_{2}$ spacer layer onto which the probed $\mathrm{Pd}$ sample particles are deposited, (b) SEM image showing a side view of the sensor structure, and (c) schematic drawing of the readout from the sensor. Upon hydride formation, the plasmon resonance of the sensor nanodisks changes. We monitor the shift of the peak position $\left(\Delta \lambda_{\text {centroid }}\right)$, change in peak height $(\Delta \mathrm{Ext})$, and/or change in the full width at half maximum of the peak $(\Delta \mathrm{FWHM})$. 

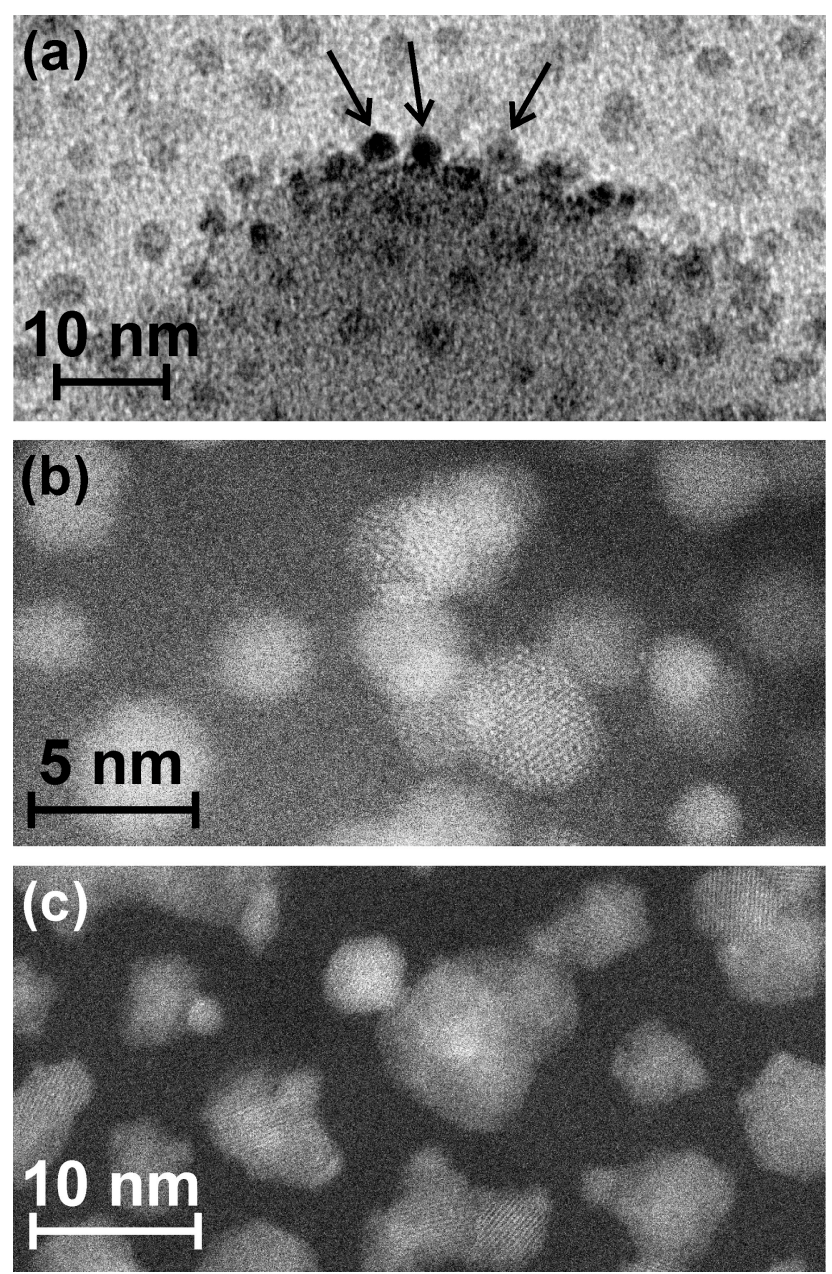

Figure 2: Tilted and high resolution TEM images of the Pd particles. (a) $\mathrm{SiO}_{2}$ cone with $\mathrm{Pd}$ particles at $30^{\circ}$ tilt angle. Particles at the edge of the cone structure (particles indicated with arrows) are seen from the side at this angle. Note the spherical shape of the particles. (b) High resolution STEM image of particles on the same sample as seen in (a). These small particles $(2-4 \mathrm{~nm})$ are single crystals. They appear to be overlapping due to the tilt angle but were confirmed to be isolated by following them during tilting. (c) Top view STEM image of larger $\mathrm{Pd}$ particles. At this size the particles take more complex shapes and are polycrystalline. 


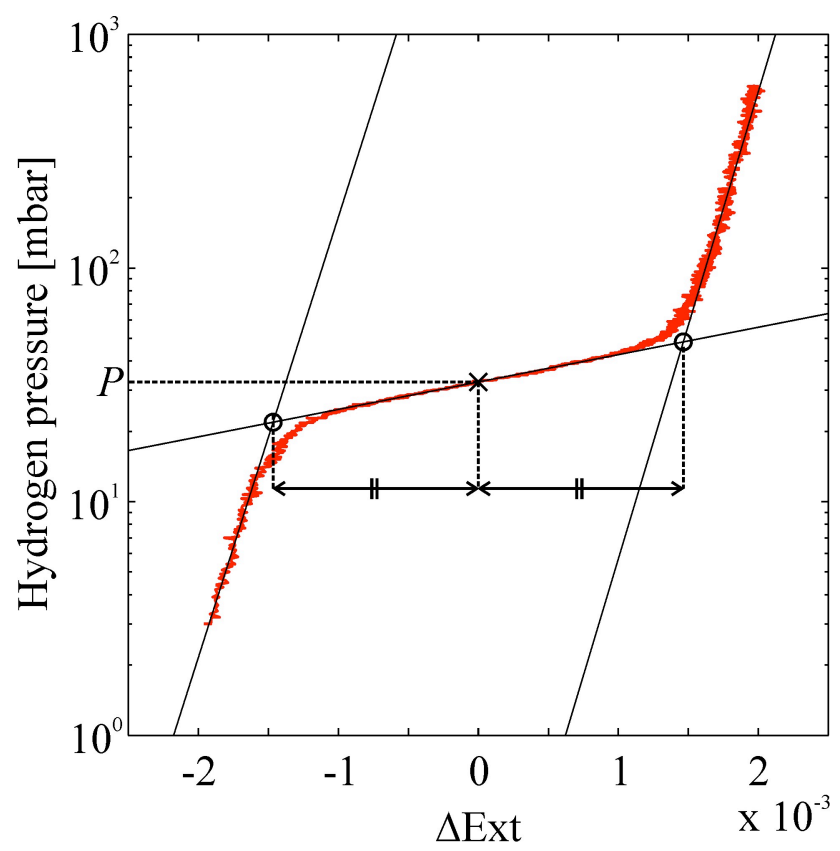

Figure 3: Typical absorption isotherm measured on Pd nanoparticles with average diameter of $3.9 \mathrm{~nm}$. $\Delta$ Ext is used as readout proportional to the concentration of absorbed hydrogen. The procedure used to find the plateau pressure $P$ from an isotherm is described in the text. The two segments marked by arrows are of equal length. 

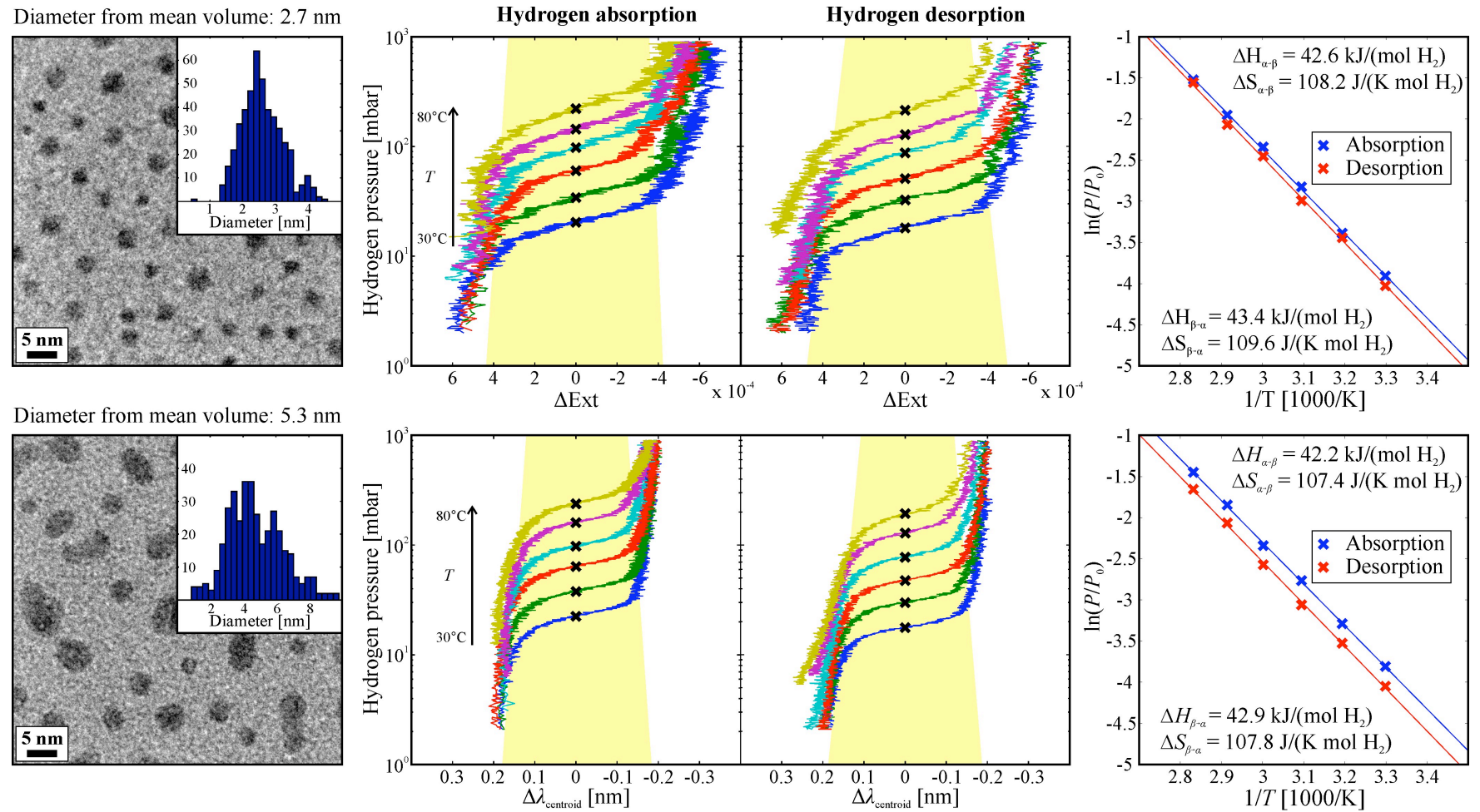

Figure 4: Experimental data for two samples with different average Pd nanoparticle sizes $(2.7 \mathrm{~nm}$ and $5.3 \mathrm{~nm})$. The left panels show TEM images and size distributions of Pd particles. The plots in the middle show hydrogen absorption and desorption isotherms for temperatures ranging from $30^{\circ} \mathrm{C}$ to $80^{\circ} \mathrm{C}$ in steps of $10^{\circ} \mathrm{C}$ (the yellow shaded area indicates a two phase coexistence region of the $\alpha$ and $\beta$ phases). To the right are Van't Hoff plots used to determine $\Delta H$ and $\Delta S$ for the hydride formation/decomposition (the obtained values for the corresponding thermodynamic parameters are shown). 


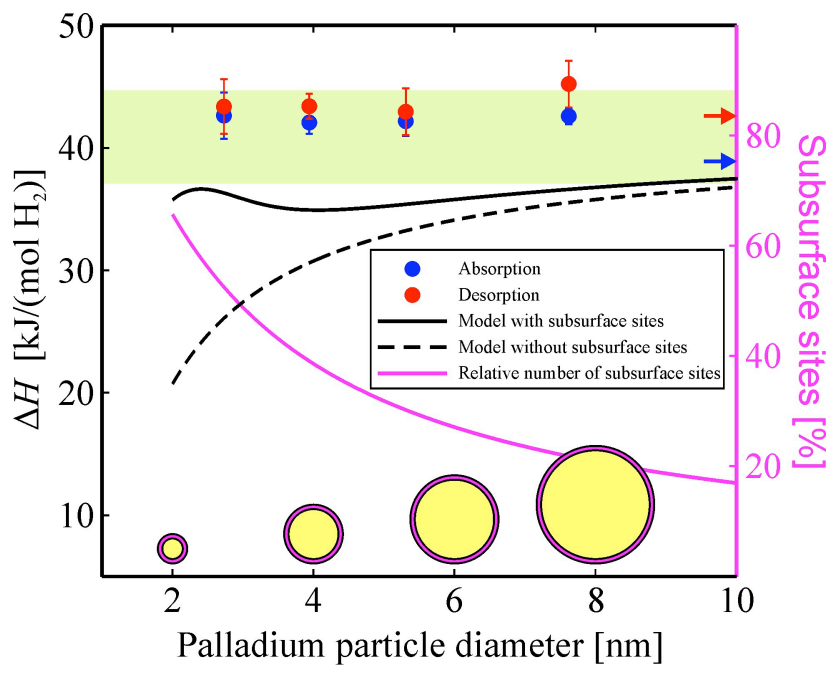

Figure 5: Size dependence of $\Delta H$ for hydride formation and decomposition in supported Pd nanoparticles. The filled circles correspond to the experimental data. The solid black line is predicted by a model including competing contributions from surface tension and hydrogen atoms occupying subsurface sites with larger absorption energies compared to bulk sites. The dashed line shows the model prediction without taking the subsurface sites into account. The arrows are experimental values for a $30 \mathrm{~nm}$ thick Pd film (see Supporting Information). The green area indicates the range of $\Delta H$ as estimated from a bulk measurement (see main text and Figure 6). The solid magenta line indicates the relative number of subsurface sites compared to the total number of bulk and subsurface sites. Also schematically shown is the proportional cross section of the nanoparticles including the subsurface volume. 


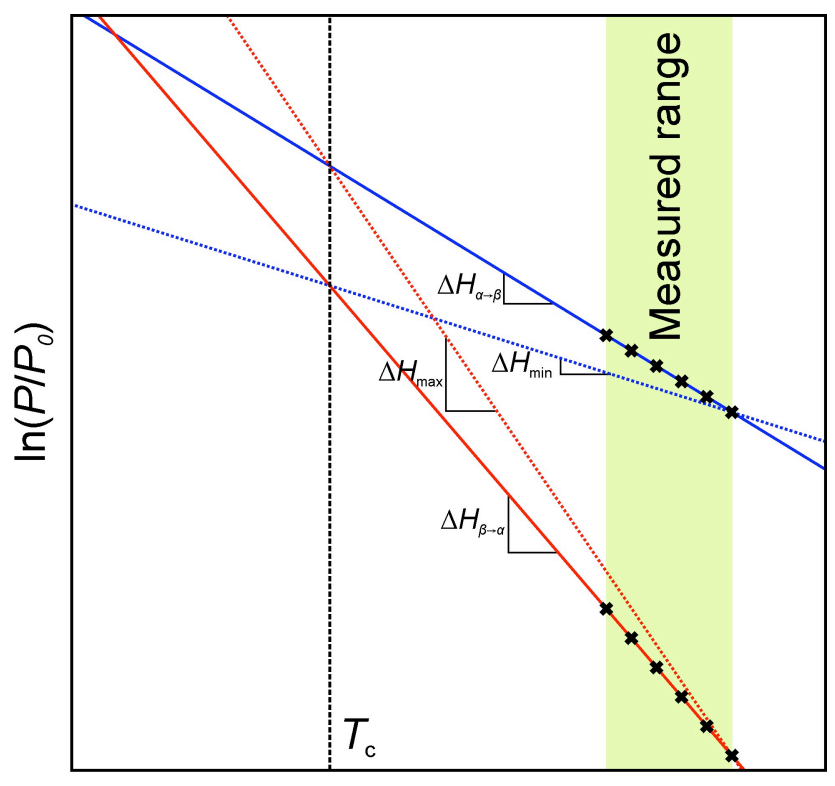

$1 / T$

Figure 6: Sketch of the procedure to estimate the possible range of the "true" $\Delta H$ from a Van't Hoff plot of a bulk measurement using the critical temperature $T_{C}$. The maximum value of $\Delta H$ is determined from the slope of a line between the point where the absorption line crosses the critical temperature and the point on the desorption line at the lowest temperature used in the measurement. The minimum is similarly determined but using the crossing between the desorption line and the critical temperature and the point of the absorption line at the lowest measured temperature instead. This yields a range of the possible values of $\Delta H$ that is outside the range from $\Delta H_{\alpha \rightarrow \beta}$ to $\Delta H_{\beta \rightarrow \alpha}$. Note that this figure is a sketch (not an actual measurement) and the effect has been exaggerated for clarity. 
Table of Contents Image

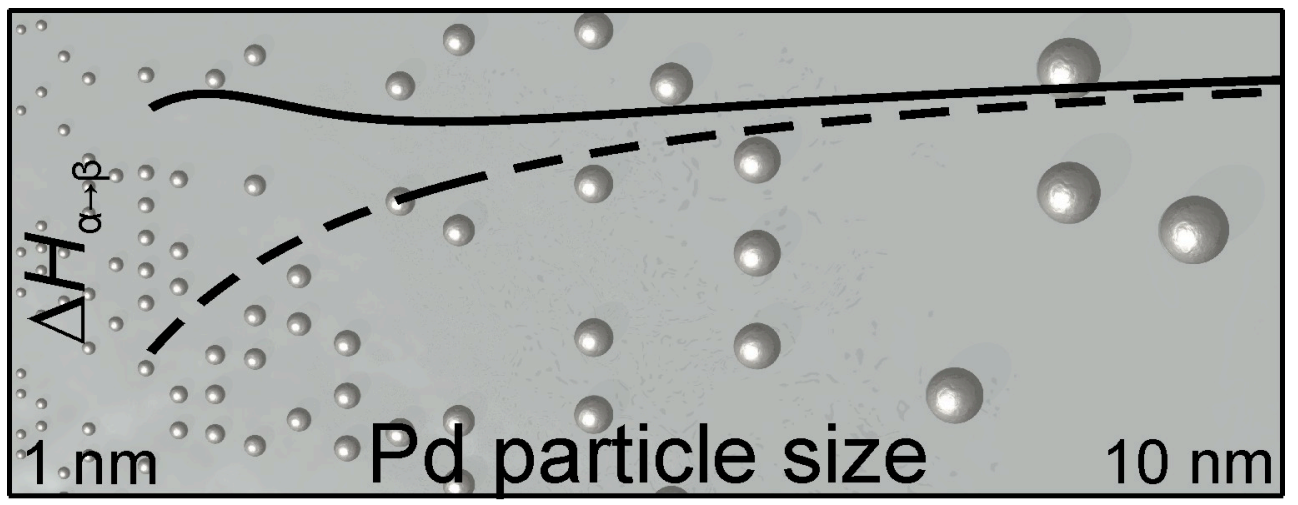

